\title{
Boundary conditions for high-shear grain flows
}

\author{
By K. HUI, P. K. HAFF, J. E. UNGAR \\ Division of Physics, Mathematics, and Astronomy, California Institute of Technology, \\ Pasadena, California 91125 \\ AND R. JACKSON† \\ Division of Chemistry and Chemical Engineering, California rostitute of Technology, \\ Pasadena, California 91125
}

(Received 3 January 1984)

\begin{abstract}
Boundary conditions are developed for rapid granular flows in which the rheology is dominated by grain-grain collisions. These conditions are $\bar{v}_{0}=$ const $\mathrm{d} \bar{v}_{0} / \mathrm{d} y$ and $u_{0}=$ const $\mathrm{d} u_{0} / \mathrm{d} y$, where $\bar{v}$ and $u$ are the thermal (fluctuation) and flow velocities respectively, and the subscript indicates that these quantities and their derivatives are to be evaluated at the wall. These boundary conditions are derived from the nature of individual grain-wall collisions, so that the proportionality constants involve the appropriate coefficient of restitution $e_{\mathrm{w}}$ for the thermal velocity equation, and the fraction of diffuse (i.e. non-specular) collisions in the case of the flow-velocity equation. Direct application of these boundary conditions to the problem of Couette-flow shows that as long as the channel width $h$ is very large compared with a grain diameter $d$ it is permissible to set $\bar{v}=0$ at the wall and to adopt the no-slip condition. Exceptions occur where $d / h$ is not very small, when the wall is not rough, and when the grain-wall collisions are very elastic. Similar insight into other flows can be obtained qualitatively by a dimensional analysis treatment of the boundary conditions. Finally, the more difficult problem of self-bounding fluids is discussed qualitatively.
\end{abstract}

\section{Introduction}

Recent advances in the description of high-shear-rate grain flows, in which the constituent particles are sensibly dispersed from one another, have been made by a number of authors (Ogawa 1978; McTigue 1978; Jenkins \& Cowin 1979; Ogawa, Umemura \& Oshima 1980; Savage \& Jeffrey 1981; Jenkins \& Savage 1981, 1983; Ackermann \& Shen 1982; Shen \& Ackermann 1982; Campbell \& Brennen 1982; Haff 1983; Hui \& Haff 1984). In this paper we borrow from the results of Haff, who obtained explicit solutions for flow patterns in Couette flow cells (Haff 1983) and in vertical channels (Hui \& Haff 1984). These solutions were derived from the basic equations for the conservation of mass,

$$
\frac{\partial \rho}{\partial t}+\frac{\partial}{\partial x_{i}}\left(\rho u_{i}\right)=0
$$

the conservation of momentum

$$
\frac{\partial}{\partial t}\left(\rho u_{i}\right)=-\frac{\partial}{\partial x_{k}}\left[p \delta_{i k}+\rho u_{i} u_{k}-\eta\left(\frac{\partial u_{i}}{\partial x_{k}}+\frac{\partial u_{k}}{\partial x_{i}}\right)\right]+\rho g_{i},
$$

$\dagger$ Present address: Department of Chemical Engineering, Princeton University, Princeton, New Jersey 08544. 
and the conservation of energy

$$
\frac{\partial}{\partial t}\left(\frac{1}{2} \rho u^{2}+\frac{1}{2} \rho \bar{v}^{2}\right)=-\frac{\partial}{\partial x_{k}}\left[\rho u_{k}\left(\frac{p}{\rho}+\frac{1}{2} u^{2}+\frac{1}{2} \bar{v}^{2}\right)-u_{i} \eta\left(\frac{\partial u_{i}}{\partial x_{k}}+\frac{\partial u_{k}}{\partial x_{i}}\right)-\kappa \frac{\partial}{\partial x_{k}} \frac{1}{2} \rho \bar{v}^{2}\right]+\rho u_{i} g_{i}-I \text {. }
$$

Here $\rho$ is the bulk density of the granular fluid, $u_{i}$ a component of the flow velocity, $p$ the pressure, $\eta$ the coefficient of viscosity, $g_{i}$ a component of the gravitational acceleration, $\bar{v}$ the grain fluctuation or 'thermal' velocity, $\kappa$ the coefficient of 'thermal' diffusivity, and $I$ the energy sink due to the fact that grain-grain collisions are always inelastic.

These equations must be augmented by supplying appropriate constitutive relationships. In a simple cell model of the granular fluid these relationships are as follows. The pressure $p$ is related to the thermal velocity $\bar{v}$ by

$$
p=t d \rho \frac{\vec{v}^{2}}{s}
$$

where $t$ is a dimensionless constant of order unity, $d$ is the grain diameter, and $s$ is the mean free path between collisions. Here we specialize to a study of dispersed $(s>0)$ but still relatively dense flows where the collisional mean free path does not exceed the grain diameter:

$$
s \ll d \text {. }
$$

This condition is thought to be typical of many possibly flow patterns, and when satisfied it leads to a considerable simplification of the laws of motion for the granular assembly.

The viscosity $\eta$ and the thermal diffusivity $\kappa$ of the granular fluid are given by

$$
\eta=q d^{2} \rho \frac{\bar{v}}{s}
$$

and

$$
\kappa=r d^{2} \frac{\bar{v}}{s}
$$

where $r$ and $q$ are dimensionless constants of order unity. Note that unlike in many standard fluid-mechanical problems $\eta$ and $\kappa$ are not fixed constants, but rather their value at any point in the granular fluid depends upon the thermal velocity (and through $s$ the density), and thus upon the solutions of the equations of motion.

Finally, the energy-sink term can be written as

$$
I=\gamma \rho \frac{\bar{v}^{3}}{s}
$$

where $\gamma$ is a parameter proportional to $1-e^{2}$, and $e$ is the coefficient of restitution describing a typical grain-grain collision. Thus $\gamma=0$ corresponds to perfectly elastic collisions.

The solutions for the flow velocity $u$, the thermal velocity $\bar{v}$, the mean free path $s$ (or the density) and the pressure $p$ can be obtained from the above equations once suitable boundary conditions are specified. Hitherto there has existed no reliable scheme for identifying such boundary conditions. In the subsequent discussion we derive plausible boundary relations for both the thermal velocity $\bar{v}$ and the flow velocity $\bar{u}$, and then, using the example of a Couette flow geometry, illustrate the way in which the solutions depend upon these conditions. 


\section{The boundary conditions}

Previously in deriving solutions to the above set of equations a standard no-slip boundary condition on the flow velocity at a rigid wall was invoked (Haff 1983; Hui \& Haff 1984). Intuitively one feels that, if the walls are sufficiently rough, slip ought to be minimized. The question of the appropriate boundary condition on the thermal velocity is less easy to think about. Haff (1983) assumed in Couette flow that the bounding walls could be made sufficiently 'soft' that $\bar{v} \approx 0$ was a good approximation there. While there is little doubt that flow containers sufficiently rough and soft to produce the desired boundary value can be designed, one would like to have in hand a general method for predicting the values of $\bar{v}$ and $u$ at a boundary. Evidently, then, the problem is not, in general, to specify values for these equations at a wall, but to specify general relations involving these quantities (and their derivatives) that can then be used together with the equations of motion and the constitutive relations to generate completely determined solutions to the flow problem.

Consider first the thermal velocity $\bar{v}$. The value that $\bar{v}$ ultimately assumes at a container wall clearly depends upon the nature of the collisions between a grain and the wall. Let each such collision be characterized by a coefficient of restitution $e_{\mathrm{w}}$. The rate of energy loss to the wall per unit area due to collisions is therefore

$$
Q_{\mathrm{w}}=a \frac{1}{2} m \vec{v}^{2}\left(1-e_{\mathrm{w}}^{2}\right) \frac{1}{d^{2}} \frac{\bar{v}}{s}
$$

where $a$ is a dimensionless constant of order unity and $m$ is the grain mass. But the energy flux conducted to the wall by virtue of grain-grain collisions is

$$
Q=\kappa \frac{\mathrm{d}}{\mathrm{d} y}\left(\frac{1}{2} \rho \vec{v}^{2}\right),
$$

where $\kappa$ is the thermal diffusivity (7) and $y$ measures distances perpendicular to the wall. Equating these two quantities leads to the equation

$$
\bar{v}_{0}=\frac{2 r d}{a\left(1-e_{\mathrm{w}}^{2}\right.} \frac{\mathrm{d} \bar{v}_{0}}{\mathrm{~d} y}
$$

where $\bar{v}_{0}$ and $\mathrm{d} \bar{v}_{0} / \mathrm{d} y$ refer to the value of the thermal velocity and its normal derivative at the wall in question. We have used the approximation $\rho \sim m / d^{3}$, valid when $s \ll d$. Equation (11) is the appropriate boundary condition on the thermal velocity.

The corresponding condition on the flow velocity can be derived in a similar way. The lateral momentum flux transmitted to the wall by grain-wall collisions is

$$
\sigma_{\mathrm{w}}=\frac{c \phi m u_{\mathrm{s}}}{d^{2}} \frac{\bar{v}_{0}}{s_{0}}
$$

where $c$ is a dimensionless constant of order unity, $s_{0}$ is the mean free path at the wall and $u_{\mathrm{s}}$ is the amount of slip there; i.e. in terms of the flow velocity $u(0)$ at the wall, and the wall velocity $u_{\mathrm{w}}$ itself,

$$
u_{\mathrm{s}}=u(0)-u_{\mathrm{w}} .
$$

The quantity $\phi$, which ranges between zero and unity, measures the fraction of collisions that transfer a significant amount of lateral momentum to the wall. 
The shear stress in the granular fluid is $\sigma=\eta \mathrm{d} u / \mathrm{d} y$, and using this in (12) together with the expression for the viscosity $\eta$ in (6) yields

$$
u_{\mathrm{s}}=\frac{q d}{c \phi} \frac{\mathrm{d} u_{0}}{\mathrm{~d} y}
$$

where $\mathrm{d} u_{0} / \mathrm{d} y$ is the normal derivative of the flow velocity evaluated at the wall. Note that $\phi$ is essentially a measure of the roughness of the wall. If $\phi$ is small so that most collisions are nearly specular, corresponding to a smooth wall, then the amount of slip $u_{\mathrm{s}}$ may be relatively large. For a rough surface, however, nearly every grain-wall collision will provide a significant transfer of lateral momentum to the wall; thus in this case $\phi$ is near unity, and the amount of slip at the wall is minimized. Equation (14) taken together with (13) provides the basic boundary condition governing the flow velocity. It is very similar to the relation first given by Maxwell (1879) in his derivation of the standard no-slip boundary condition for ordinary fluids.

\section{Applications to Couette flow}

Let us consider the flow in a Couette cell consisting of an upper plate moving in the positive $x$-direction with velocity $\Delta u$ and a fixed lower plate. Defining the dimensionless thermal velocity

and dimensionless height

$$
V \equiv \frac{\bar{v}}{\Delta u}
$$

$$
Y \equiv \frac{y}{h},
$$

the energy equation (3) becomes

Here

$$
\frac{\mathrm{d}^{2} V}{\mathrm{~d} Y^{2}}+\Omega^{2} V=0
$$

$$
\Omega^{2} \equiv \frac{t^{2}}{q r \delta^{2}} \frac{\Sigma_{0}^{2}}{P_{0}^{2}}-\frac{\gamma}{r \delta^{2}},
$$

where

$$
\Sigma_{0} \equiv \sigma_{0} / \rho d^{2}\left(\frac{\Delta u}{h}\right)^{2}
$$

is the dimensionless shear stress,

$$
P_{0} \equiv p_{0} / \rho d^{2}\left(\frac{\Delta u}{h}\right)^{2}
$$

is the dimensionless pressure, and

$$
\delta \equiv d / h
$$

is the dimensionless grain diameter. We note that the ratio $\Delta u / h$ appearing in the definitions of $\Sigma_{0}$ and $P_{0}$ is the rate of shear, as judged by the moving boundaries of the Couette cell.

By the symmetry of the Couette cell about the plane $Y=\frac{1}{2}$, we may immediately write down the solution to (15) as

$$
V=B \cos \Omega\left(Y-\frac{1}{2}\right),
$$

where $B$ is a not-yet-determined dimensionless constant. Rewriting the boundary condition (11) in the dimensionless form

$$
V(0)=\frac{2 r \delta}{a\left(1-e_{\mathrm{w}}^{2}\right)}\left(\frac{\mathrm{d} V}{\mathrm{~d} Y}\right)_{0},
$$


we find upon substituting (17) that

$$
\frac{2 r \delta}{a\left(1-e_{\mathrm{w}}^{2}\right)} \Omega=\cot \frac{1}{2} \Omega .
$$

The $x$-component of the momentum equation (2), combined with the definitions (4) and (6) and suitably non-dimensionalized, becomes

$$
\frac{\mathrm{d} U}{\mathrm{~d} Y}=\frac{t}{q \delta} \frac{\Sigma_{0}}{P_{0}} V
$$

which upon substitution of $V$ from (17) becomes

$$
\frac{\mathrm{d} U}{\mathrm{~d} Y}=B \frac{t}{q \delta} \frac{\Sigma_{0}}{P_{0}} \cos \Omega\left(Y-\frac{1}{2}\right)
$$

where the dimensionless flow velocity has been defined by

One may integrate (19) to derive

$$
U=\frac{u}{\Delta u} \text {. }
$$

$$
U(Y)-U(0)=\frac{t}{q} \frac{1}{\delta} \frac{\Sigma_{0}}{P_{0}} \frac{B}{\Omega}\left[\sin \Omega\left(Y-\frac{1}{2}\right)+\sin \frac{1}{2} \Omega\right]
$$

In particular,

$$
U(1)-U(0)=2 \frac{B}{\Omega} \frac{t}{q \delta} \frac{\Sigma_{0}}{P_{0}} \sin \frac{1}{2} \Omega .
$$

The dimensionless version of the 'slip' boundary condition (14) gives

$$
U(0)=\frac{q}{c} \frac{\delta}{\phi}\left(\frac{\mathrm{d} U}{\mathrm{~d} Y}\right)_{0}=\frac{q}{c} \frac{\delta}{\phi} B \frac{t}{q \delta} \frac{\Sigma_{0}}{P_{0}} \cos \frac{1}{2} \Omega
$$

and

$$
1-U(1)=\frac{q}{c} \frac{\delta}{\phi}\left(\frac{\mathrm{d} U}{\mathrm{~d} Y}\right)_{1}=\frac{q}{c} \frac{\delta}{\phi} B \frac{t}{q \delta} \frac{\Sigma_{0}}{P_{0}} \cos \frac{1}{2} \Omega
$$

(where we note that the dimensionless velocity of the top plate is 1 ). Combining (18) and (21)-(23), we find for the normalization constant $B$

giving us

$$
\frac{1}{B}=\frac{2}{\Omega} \frac{t}{q \delta} \frac{\Sigma_{0}}{P_{0}} \sin \frac{\Omega}{2}\left[1+\frac{q \delta}{c \phi} \frac{2 r \delta}{a\left(1-e_{\mathrm{w}}^{2}\right)} \Omega^{2}\right],
$$

and

$$
U(Y)=\frac{1}{2}\left\{1+\frac{\sin \Omega\left(Y-\frac{1}{2}\right)}{\sin \frac{\Omega}{2}\left[1+\frac{q \delta}{c \phi} \frac{2 r \delta}{a\left(1-e_{\mathrm{w}}^{2}\right)} \Omega^{2}\right]}\right\}
$$

$$
V(Y)=\frac{\cos \Omega\left(Y-\frac{1}{2}\right)}{\frac{2}{\Omega} \frac{t}{q \delta} \frac{\Sigma_{0}}{P_{0}} \sin \frac{\Omega}{2}\left[1+\frac{q \delta}{c \phi} \frac{2 r \delta}{a\left(1-e_{\mathrm{w}}^{2}\right)} \Omega^{2}\right]} .
$$

Equation (16) determines the ratio

$$
\frac{\sigma_{0}}{p_{0}}=\frac{\Sigma_{0}}{P_{0}}=(q r)^{\frac{1}{2}} \frac{\delta \Omega}{t}\left(1+\frac{\gamma}{r \delta^{2} \Omega^{2}}\right)^{\frac{1}{2}},
$$

which can be substituted into (26) yielding

$$
V(Y)=\frac{\cos \Omega\left(Y-\frac{1}{2}\right)}{2\left(\frac{r}{q}\right)^{\frac{1}{2}}\left(1+\frac{\gamma}{r \delta^{2} \Omega^{2}}\right)^{\frac{1}{2}} \sin \frac{\Omega}{2}\left[1+\frac{q \delta}{c \phi} \frac{2 r \delta}{a\left(1-e_{\mathrm{w}}^{2}\right)} \Omega^{2}\right]}
$$


The pressure $P$ is found as follows. From the mass-conservation requirement

$$
\int_{0}^{h} \rho(y) \mathrm{d} y=\int_{0}^{h-\Delta h} \rho_{0} \mathrm{~d} y
$$

where $\rho_{0}$ is the density of grains when they are packed together most closely and $\Delta h$ the resulting 'free-space' parameter in the Couette cell, it can be shown (Haff 1983) that

$$
\frac{\Delta h}{h}=\frac{3}{\delta} \int_{0}^{1} \frac{s}{h} \mathrm{~d} Y .
$$

Since $\Delta h$ is presumed to be known for the flow in question (it measures the 'fullness' of the Couette cell), we can combine (27) with the equation of state (4) to give

$$
P_{0}=\frac{3 t}{\delta^{2}} \frac{h}{\Delta h} \int_{0}^{1} V^{2} \mathrm{~d} Y .
$$

Combining with (28), this yields

$$
P_{0}=\frac{3}{8} \frac{h}{\Delta h} \frac{q t}{r \delta^{2}} \frac{1}{\left(1+\frac{\gamma}{r \delta^{2} \Omega^{2}}\right)} \frac{\left[\frac{\Omega+\sin \Omega}{\Omega \sin ^{2} \frac{1}{2} \Omega}\right]}{\left[1+\frac{q \delta}{c \phi} \frac{2 r \delta}{a\left(1-e_{\mathrm{w}}^{2}\right)} \Omega^{2}\right]^{2}}
$$

which can in turn be combined with (16) to give

$$
\Sigma_{0}=\frac{3}{8} \frac{h}{\Delta h} \frac{q^{\frac{3}{2}}}{r^{\frac{1}{2} \delta}} \frac{1}{\left(1+\frac{\gamma}{r \delta^{2} \Omega^{2}}\right)^{\frac{1}{2}}} \frac{\left[\frac{\Omega+\sin \Omega}{\sin ^{2} \frac{1}{2} \Omega}\right]}{\left[1+\frac{q \delta}{c \phi} \frac{2 r \delta}{a\left(1-e_{\mathrm{w}}^{2}\right)} \Omega^{2}\right]^{2}}
$$

In performing a measurement on Couette flow in a cell of given height $h$, with fixed values of the parameters that specify the properties of the grains and cell walls, namely $q, r, t, \gamma, e_{\mathrm{w}}, a, c, \phi$ and $d$, the experimenter may choose to vary any of four parameters: $\Delta u, \Delta h, \sigma_{0}$ and $p_{0}$. Of these four parameters, only two at a time can in fact be varied independently; if, for example, the 'fullness' of the cell is specified (as measured by the value of $\Delta h$ ), and the upper plane is pulled until it moves at a specified velocity $\Delta u$, it is clear on physical grounds that the experiment has been specified completely, and that there is a unique stress $\sigma_{0}$ with which the upper plane must be pulled to bring it up to velocity $\Delta u$, and these is similarly a unique value of the pressure $p_{0}$ that will be exerted by the fluid on the top and bottom plates of the cell. This fact is also evident from the preceding mathematical analysis; by (18) the values of $d, h, a, r$ and $e_{\mathrm{w}}$ determine $\Omega$, which in turn determines the flow and thermal velocity patterns, as well as the pressure and shear stress by (25), (26), (30) and (31) respectively. Similarly, the experimenter may fix $p_{0}$ and $\Delta h$ by filling the cell to the degree specified by $\Delta h$ and then pulling on the upper plate with increasing stress $\sigma_{0}$ until the pressure measured inside the cell rises to $p_{0}$, at which time the upper plate will be moving at some well-defined velocity $\Delta u$. In fact, one may choose to specify the experimental conditions by any one of the following five pairs of parameters: $(\Delta u, \Delta h),\left(\Delta u, \sigma_{0}\right),\left(\Delta u, p_{0}\right),\left(\Delta h, \sigma_{0}\right)$ and $\left(\Delta h, p_{0}\right)$.

One may ask whether one can specify the experimental conditions by the parameter pair $\left(\sigma_{0}, p_{0}\right)$; let the experimenter pull on the top plate with force per unit area $\sigma_{0}$ and add grains to the Couette cell (i.e. change $\Delta h$ ) until the pressure reaches the desired value of $p_{0}$. In fact, the eigenvalue equation (18), together with the defining equation (16) for $\Omega$, shows that one may not specify both $\sigma_{0}$ and $p_{0}$ (or equivalently 
$\Sigma_{0}$ and $P_{0}$ ); steady-state solutions exist only for the ratio fixed by (27). In physical terms, (18) determines the ratio of shear stress to pressure necessary so that the vibrational energy generated by viscous dissipation exactly balances the energy lost to inelasticities in grain-wall and grain-grain collisions. If the experimenter imposes $\sigma_{0}$ and $p_{0}$ such that

$$
\frac{\sigma_{0}}{p_{0}}>(q r)^{\frac{1}{2}} \frac{\delta \Omega}{t}\left(1+\frac{\gamma}{r \delta^{2} \Omega^{2}}\right)^{\frac{1}{2}}
$$

there is no mechanism by which the vibrational energy being generated can be dissipated, and therefore no steady state can be found; instead $\bar{v}$ will increase without bound as time progresses. Because, for specified $p_{0}, \bar{v}$ and $s$ are related by (4), $s$ (or equivalently $\Delta h$ ) will also grow as $\vec{v}^{2}$. Of course, when $s$ becomes large enough that the condition $s \ll d, h$ breaks down, the continuum assumption is rendered invalid.

In reality, the coefficients of restitution for both grain-grain and grain-plane collisions are slowly decreasing functions of the impact velocity (Goldsmith 1960), and therefore as more energy is fed into the fluid and $\bar{v}$ grows, $\gamma$ and (by (18)) $\Omega$ grow as well, which by (27) shows that a steady state can be found even for $\sigma_{0} / p_{0}$ larger than that calculated using the coefficients of restitution evaluated at low impact velocity. In practice, however, the decrease of the coefficient of restitution with increasing impact velocity is so slow that the continuum assumption breaks down before steady state can be achieved, except for cases in which $\sigma_{0} / p_{0}$ is very close to satisfying (27).

If on the other hand, the ratio of $\sigma_{0}$ to $p_{0}$ is smaller than required by (27), the rate at which energy is being pumped into the vibrational degree of freedom of the fluid by viscous dissipation is too low to make up for the inelastic losses at the boundaries and within the fluid itself, and therefore both $\bar{v}$ and $s$ (or equivalently $\Delta h$ ) must fall with time. When $s$ becomes very small (the exact value being determined by the shape of the grains), each grain makes contact with several neighbours at the same time, and the binary-collision hypothesis that underlies the present model breaks down. Grain flow in this 'high-density' regime remains to be investigated; it is to be expected, however, that when the ratio of pressure to shear stress becomes sufficiently large, the medium will seize up into a stationary, solid-like mass.

\section{Discussion of Couette-flow results}

It is instructive to discuss in greater detail the behaviour of the Couette-flow solutions. This is best done by studying several limiting cases. The continuum requirement decrees that $\delta \ll 1$, and therefore, unless the grain-wall interaction is almost perfectly elastic,

we have

$$
\begin{aligned}
& 1-e_{\mathrm{w}}^{2} \ll 1, \\
& \frac{2 r \delta}{a\left(1-e_{\mathrm{w}}^{2}\right)} \ll 1,
\end{aligned}
$$

and by (18) $\Omega \approx \pi$. If in addition we restrict attention to walls that are not very smooth and grains that are not very elastic (i.e. we exclude $\phi, \gamma \approx 0$ ), then $q \delta / c \phi \leqslant 1$ and $\gamma / r \delta^{2} \Omega^{2} \gg 1$. In this limit (25), (26), (30) and (31) reduce respectively to

$$
\begin{gathered}
U(Y)=\sin ^{2} \frac{1}{2} \pi Y, \\
V(Y)=\frac{\pi q^{\frac{1}{2}} \delta \sin \pi Y,}{2 \gamma^{\frac{1}{2}}}, \\
P_{0}=\frac{3 \pi^{2}}{8} \frac{h}{\Delta h} \frac{q t}{\gamma}
\end{gathered}
$$


and

$$
\Sigma_{0}=\frac{3 \pi^{2}}{8} \frac{h}{\Delta h} \frac{q^{\frac{3}{2}}}{\gamma^{\frac{1}{2}}}
$$

These are identical with the results obtained (Haff 1983) using the simplified boundary conditions

$$
V(0)=V(1)=0
$$

on the thermal velocity, together with the no-slip conditions

$$
U(0)=0, \quad U(1)=1
$$

on the flow velocity. We therefore conclude that the simplified boundary conditions $(37)-(39)$ are valid whenever the continuum assumption $(\delta \ll 1)$ is, except when the grain-grain or grain-plain collisions are almost perfectly elastic, or when the grain-plane collisions are almost perfectly specular.

In the opposite limit, for which the grain-plane collisions are almost perfectly elastic,

$$
1-e_{\mathrm{w}}^{2} \ll \delta,
$$

(18) shows that $\Omega=0$. More accurately, in this limit (18) becomes

$$
\Omega^{2}=\frac{a\left(1-e_{\mathrm{w}}^{2}\right)}{r \delta} .
$$

Let us suppose that the grain-grain collisions too are highly elastic

Then we have

$$
\gamma \ll 1 \text {. }
$$

$$
\frac{\gamma}{r \delta^{2} \Omega^{2}} \approx \frac{\gamma}{\delta a\left(1-e_{\mathrm{w}}^{2}\right)} \gg 1
$$

Equations (25), (26), (30) and (31) reduce respectively to

and

$$
\begin{aligned}
& U(Y)=\frac{1}{2}\left[1+\frac{2 Y-1}{1+2 \frac{q \delta}{c \phi}}\right], \\
& V(Y)=\frac{q^{\frac{1}{2}} \delta}{\gamma^{\frac{1}{2}}\left(1+2 \frac{q \delta}{c \phi}\right)}, \\
& P_{0}=3 \frac{h}{\Delta h} \frac{q t}{\gamma} \frac{1}{\left(1+2 \frac{q \delta}{c \phi}\right)^{2}}
\end{aligned}
$$

$$
\Sigma_{0}=3 \frac{h}{\Delta h} \frac{q^{\frac{3}{2}}}{\gamma^{\frac{1}{2}}} \frac{1}{\left(1+2 \frac{q \delta}{c \delta}\right)^{2}} .
$$

It is notable that (43) predicts that the thermal velocity is independent of position within the Couette cell, and (42) shows that the flow velocity gradient is independent of position. This behaviour is also exhibited by ordinary Newtonian fluids in a Couette cell. This similarity is not surprising, in view of the elastic character of molecular collisions.

We may further specialize the results in the elastic limit to the non-specular limit

$$
\frac{q \delta}{c \phi} \ll 1,
$$


for which the flow-velocity equation (42) goes to

$$
U(Y)=Y \text {. }
$$

Clearly, the no-slip boundary condition is satisfied. Equation (43) describing the thermal-velocity distribution becomes

$$
V(Y)=\frac{q^{\frac{1}{2}} \delta}{\gamma^{\frac{1}{2}}}
$$

showing that the thermal velocity does not go to zero at the boundaries.

\section{General discussion of boundary conditions}

The Couette-flow example of $\$ 33$ and 4 gives a clear illustration of the application of the boundary conditions (11) and (14) to a specific problem. It is useful, however, to make some observations of a more general nature.

In any type of rapid granular-flow problem let $V_{T}$ be a typical value of the thermal velocity $\bar{v}$ and let $D$ be a typical distance over which $\bar{v}$ changes significantly. Then (11) is roughly equivalent to the requirement that

$$
\bar{v}_{\mathbf{0}} \sim \frac{1}{1-e_{\mathrm{w}}^{2}} \frac{d}{D} V_{\mathrm{T}}
$$

For grains whose collisions with the walls are not too elastic, it is appropriate to set $\bar{v}_{0}=0$ at the boundary as long as $d / D \ll 1$. In the Couette-flow example, $D \approx h$, and so setting the thermal velocity to zero at the plates is justified as long as the channel has a width of many grain diameters.

Similar remarks pertain to the flow-velocity boundary condition (14). If $W$ is a typical value of the flow velocity $u$, and $D$ is the distance over which $u$ changes significantly then

$$
u_{\mathrm{s}} \sim \frac{1}{\phi} \frac{d}{D} W
$$

As above, provided that the fraction $\phi$ of diffuse collisions is not too small, then the amount of slip at a boundary is small if $d \ll D$, as is true in Couette flow. Equation (14), and the general argument leading to (49), are very similar to results obtained originally by Maxwell (1879) in his derivation of the no-slip condition.

We note that in principle there are experiments which can be performed at the microscopic (i.e. granular) level to shed light on the coefficients appearing in (11) and (14). Just as in the bulk material the important quantity $e$, the coefficient of restitution in grain-grain collisions, can be determined independently of particular flow conditions by collision experiments with individual grains, so in the case of grain-wall collisions the corresponding quantities $e_{\mathrm{w}}$ and $\phi$ depend upon the properties of individual grain-wall collisions, which can be investigated experimentally.

In the simplest model of energy loss, it would suffice to measure the change in the normal velocity component in single grain-wall collisions to determine $e_{w}$. The results of these measurements would depend upon the composition of the wall (and of the grain), but would be essentially independent of the wall shape.

To get an idea of the value of $\phi$, measurements of the change in the tangential component of the grain velocity upon collision with the wall are required. For a smooth wall, the change will be small for all collisions, and hence significant slip will occur (equation (49)). For a rough wall the change may be significant for many 
collisions, or it may be significant for only a few collisions, depending upon the nature of the roughness. Thus if the wavelength and amplitude of the roughness are comparable to the size of a grain, a large fraction of the collisions will result in significant lateral-momentum transfer to the wall, and $\phi$ will be large. However, if the roughness is due to occasional 'bumps' on the surface spaced many grain diameters apart, then most collisions will be specular and $\phi$ will be small.

Unfortunately, in the present heuristic theory, as in Maxwell's paper, it is possible to relate $\phi$ only qualitatively to grain-wall collision data, inasmuch as the correct distribution functions necessary to average the various collision possibilities are not in hand. Thus $\phi$, and to a lesser degree $e_{\mathrm{w}}$, remain as parameters which must be determined empirically until a more detailed theory is available.

We do know, however, what the necessary data base is that would be required in principle to compute $\phi$. Namely, tangential velocity changes $\Delta \bar{v}$ need to be measured for a large number of collisions of varying velocities and incident angles on a representative section of the wall. Only such velocity changes oriented along the actual flow direction will contribute to $\phi$. Thus if the wall is constructed of a series of parallel ridges which in turn are parallel to the flow velocity, little net shear stress is transmitted, even though $\Delta \bar{v}$ may be large for collisions transverse to the pattern. On the other hand, if the ridge pattern is oriented perpendicularly to the flow, then a large shear stress will be applied to the wall. In the most general case the direction of the shear stress applied to the wall will not be along the axis of the channel, but will be inclined at an angle determined by the orientation of the ridge pattern. In such cases, boundary conditions on $u$ will become more complicated and (14) is no longer sufficient to determine the flow. In general, for surfaces that exhibit a direction dependence of the roughness, a tensorial boundary condition on $u$ is required, and the simple two-dimensional flow systems considered in this paper are no longer meaningful. We do not consider this possibility further.

To conclude this section a few remarks on the roughness scale of bounding surfaces are in order. If the mean amplitude and wavelength of surface roughness are very small compared with a grain diameter then the surface can be taken to be smooth as far as calculating the shear stress is concerned. However, if the roughness is comparable to the grain size then substantial shear stresses can be generated, as discussed above.

We may then inquire about the case when the roughness scale is large in relation to the grain diameter. In this case one natural approach is simply to adopt the large-scale roughness as defining the macroscopic shape of the container, the effect of smaller-scale surface topography being retained in the boundary condition as usual. However, owing to the nature of a granular fluid, a phenomenon that can be called 'self-bounding' may occur, which in effect leads to a smoothing out of large-scale surface roughness.

Consider granular flow past a flat wall with a rectangular notch cut into it. The dimensions of the notch are supposed to be large compared with a grain diameter. If the grain-grain collisions are elastic, and the energy loss to the wall is not too large, then a circulation cell (or cells) would be set up within the notch, as in the case of a molecular fluid.

Because of the lossy nature of a granular fluid, however, such circulation cells may be incipiently damped. Consider a parcel of fluid circulating around the cavity. During the time of circulation the grains in the parcel suffer many collisions, and hence energy is lost which cannot easily be replaced from viscous heating in the main channel, except by conduction. If the channel depth $l$ is of the order of the conduction length $\lambda$ (equation (49)) then a reasonable amount of fluidization may be retained in the 
notch, and some flow will occur there. But if $l \gg \lambda$ then all grains except those within $\sim \lambda$ of the mouth of the notch will come to rest as an underforming mass. Thus the dynamics of the grains themselves leads to an effective smoothing of the plate boundary.

This must be a general phenomenon of granular fluids, namely that the existence of sharp angles in a flow path, which would require fluid-parcel trajectories to become remote from the main region of shearing (and fluidization), must be expected to lead to the formation of stagnant zones where all flow ceases, as in the example of the notch above, or in the no-flow regions often encountered in large-angle hopper flows (see e.g. Nguyen, Brennen \& Sabersky 1979). This behaviour is traceable directly to the fact that grain-grain collisions are always inelastic; such zones of complete stagnation cannot develop in ordinary fluids.

The details of a theory to predict the shape of the flow boundary formed by such self-bounding fluids have not yet been developed, but such behaviour is clearly a general property of granular fluids and deserves further attention.

We would like to thank R. L. Shreve for helpful discussions. This work was supported in part by the National Science Foundation (EAR 82-12372). During the course of this work R. Jackson was a Sherman Fairchild Distinguished Scholar at Caltech.

\section{REFERENCES}

Ackermann, N. L. \& Shen, H. 1982 Stresses in rapidly sheared fluid-solid mixtures. J. Engng Mech. Div. ASCE 108 (EM1), 95-113.

Campbell, C.S. \& Brennen, C.E. 1982 Computer simulations of chute flows of granular materials. In Deformation and Failure in Granular Materials (ed. P. A. Vermeer \& H. J. Luger). Bulkenna.

Goldsuith, W. 1960 Impact, the Theory and Physical Behaviour of Colliding Solids. Edward Arnold.

HafF, P. K. 1983 Grain flow as a fluid-mechanical phenomenon. J. Fluid Mech. 134, 401-430.

HuI, K. \& HAFF, P. K. 1984 Rapid grain flow in a vertical channel. Submitted to J. Fluid Mech.

Jenkins, J. T. \& Cowin, S. C. 1979 Theories for flowing granular materials. In Mechanics Applied to the Transport of Bulk Materials (ed. S. C. Cowin), pp. 79-89. ASME, AMD-31.

Jenkins, J. T. \& SAVAGE, S. B. 1981 The mean stress resulting from interparticle collisions in a rapid granular shear flow. In Continuum Models of Discrete Systems 4 (ed. O. Brulin \& R. K. T. Hsieh), pp. 356-371. North-Holland.

JENKINS, J. T. \& SAVActe, S. B. 1983 A theory for the rapid flow of identical, smooth, nearly elastic particles. J. Fluid Mech. 130, 187-202.

McTigue, D. F. 1978 A model for stresses in shear flow of granular material. In Proc. US-Japan Seminar on Continuum-Mechanical and Statistical Approaches in the Mechanics of Granular Materials (ed. S. C. Cowin \& M. Satake), pp. 266-271. Gakujutsu-Bunken Fukyukai.

MAXWELL, J. C. 1879 On the stresses in rarefied gases arising from inequalities of temperature. Phil. Trans. R. Soc. Lond. 170, 231-256.

Nauyen, T., Brennen, C. \& Sabersky, R. 1979 Funnel flow in hoppers. In Mechanics Applied to the Transport of Bulk Materials (ed. S. C. Cowin), pp. 25-33. ASME, AMD-31.

OGaWA, S. 1978 Multitemperature theory of granular materials. In Proc. US-Japan Seminar on Continuum-Mechanical and Statistical Approaches in the Mechanics of Granular Materials (ed. S. C. Cowin \& M. Satake), 189-207. Gakujutsu-Bunken Fukyukai.

Oqawa, S., Umemura, A. \& Oshima, N. 1980 On the equations of fully fluidized granular materials. Z. angew. Math. Phys. 31, 483-493.

SAVAGE, S. B. \& JEFFREY, D. J. 1981 The stress tensor in a granular flow at high shear rates. $J$. Fluid Mech. 110, 255-272.

Shen, H. \& Ackermann, N. L. 1982 Constitutive relationships for fluid-solid mixtures. J. Engng Mech. Div ASCE 108 (EM5), 748-763. 